\title{
De l'impression à l'expression : stylèmes du suspens(e) chez Proust
}

Geneviève Henrot Sostero

\section{OpenEdition}

\section{$\checkmark$ Journals}

Édition électronique

URL : https://journals.openedition.org/ml/4105

DOI : $10.4000 / \mathrm{ml} .4105$

ISSN : 2274-0511

Éditeur

Association Modèles linguistiques

\section{Édition imprimée}

Date de publication : 29 décembre 2017

Pagination : 79-104

Référence électronique

Geneviève Henrot Sostero, « De l'impression à l'expression : stylèmes du suspens(e) chez Proust », Modèles linguistiques [En ligne], 75 | 2017, document 4, mis en ligne le 28 novembre 2018, consulté le 01 juillet 2021. URL : http://journals.openedition.org/ml/4105; DOI : https://doi.org/10.4000/ml.4105 


\section{De l'impression à l'expression : stylèmes du suspens(e) chez Proust}

Geneviève Henrot Sostero

\section{Introduction}

Le terme $\mathrm{d}^{\prime}$ « impressionnisme » a quelques fois été associé à Proust. Divers parcours critiques affrontent la question, selon que l'orientation choisie s'attache aux faits historico-biographiques, aux relations intersémiotiques, au phénomène psychologique ou à sa traduction stylistique.

Dans l'ordre de l'histoire des Lettres et des Arts, et de la biographie d'auteur, certains érudits ont minutieusement reconstitué les rencontres possibles ou certifiées entre Proust et tel peintre ou telle toile, justifiant par là la légitimité - ou la liberté prise - d'allusions parsemées dans la fiction. Les relations de Proust avec la peinture visible à son époque n'ont plus de secrets pour Kazuyoshi Yoshikawa, qui a patiemment établi au cours d'une vie de recherche toute la biographie pictomane de Proust, depuis ses premières chroniques d'art jusqu'à sa fatale visite à l'exposition Vermeer de $1922^{1}$.

D'autres critiques axent leur comparaison moins sur la circonstance historique externe de la connaissance esthétique que sur la

1. Kazuyoshi Yoshikawa, Proust et l'art pictural, Paris, Honoré Champion, « Recherches proustiennes » n. 14, 2010. Pour une synthèse, consulter «Impressionnisme », dans Annick Bouillaguet et Brian R. Rogers (dir.), Dictionnaire Marcel Proust, Paris, Honoré Champion, 2004, 2014²: 496-498. Sur le même sujet, Maurice Eugène Chenowitz, Proust and Painting, New York, International University Press, 1945 ; Juliette Monnin-Hornung, Marcel Proust et la peinture, Genève, Droz, 1951 ; Sophie Bertho, (dir.), Proust et ses peintres, Amsterdam, CRIN 37, 2000 ; Michèle Magill, Répertoire des références aux arts et à la littérature dans À la recherche du temps perdu de Marcel Proust, Birmingham, Summa, 1991. 
plasticité même des œuvres convoquées, montrant chez Proust des intentions plus ou moins manifestes d'imitation, d'allusion, de pastiche ${ }^{2}$.

Dans une mouvance plus strictement thématique, thèmes et motifs circulent tout naturellement à une même époque, repris en fugue et variation grâce à la diversité des langages : le floréal du Modern Style n'aurait-il pas inspiré le regard qui métamorphose les jeunes filles en filles-fleurs ? ${ }^{3}$ Les nymphéas de la Vivonne n'ont-ils pas souvenir de Giverny $^{4}$, et les marines d'Elstir n'ont-elles pas pour « cartons » certains Turner ?5 Cette perspective intersémiotique installe la Recherche dans l'aura d'un « air du temps » où tous les arts dialoguent entre eux et s'échangent des vues et des saveurs, des préférences ou des résistances.

Un sondage plus formel a fait extraire, à d'autres critiques encore, la « formule esthétique » propre à Monet, à Cézanne, à Turner ou à Whistler ${ }^{6}$, pour y trouver une transposition ou des affinités, non tant de motifs que de technique ou de manière, dans certaines caractéristiques de la représentation chez Proust : jeux de lumière, dominance des notations de couleurs, importance de l'atmosphère et des visions floues qu'elle conditionne (brumes, pluies, pénombres). Telle est d'ailleurs une des premières acceptions données au diagnostic d' « impressionnisme » appliqué à des œuvres littéraires : Brunetière n'attribuait-il pas au Daudet des Rois en exil la même capacité formelle de « décrire par petites touches » qui a caractérisé l'école de Barbizon ?8

2. Pour une (rapide) synthèse, voir mon « Proust e l'impressionismo », Quaderni proustiani, $\mathrm{n}^{\circ} 10,2016: 53-70$

3. "Art nouveau", Dictionnaire Marcel Proust, op. cit., sub voce. Marion Schmid, Proust dans la décadence, Paris, Honoré Champion, « Recherches proustiennes » $\mathrm{n}^{\circ}$ 12, 2008 ; Ead., " Marcel Proust's Modernist Novel of Time », dans Michael Bell, ed., The Cambridge Companion to European Novelists, Cambridge, Cambridge University Press, 2011 : 65-79 ; Ead., « Proust et le style décadent », Bulletin d'informations proustiennes, $\mathrm{n}^{\circ} 37$ (2007) : 101-116.

4. «Monet», D Dictionnaire Marcel Proust, op. cit., sub voce.

5. Isabelle Zuber, Tableaux littéraires : Les marines dans l'cuvre de Marcel Proust, Peter Lang, 1998.

6. «Rembrandt », «Cézanne », «Whistler », Dictionnaire Marcel Proust, op. cit., sub voce. Mais d'autres styles picturaux pourraient aussi bien faire l'affaire, de Rembrandt au cubisme, tant la Recherche se présente comme une œuvre-monde.

7. Taeko Uenishi, Le Style de Proust et la peinture, Paris, SEDES, 1988. 
Ainsi, à la question de la relation entre Proust et l'impressionnisme, peut-on vouloir apporter toutes sortes de réponses.

Bien plus rares sont les critiques proustiens qui ont abordé le terme «impressionniste » hors de toute acception picturale, remontant à son étymon linguistique : " lié à l'impression première $»^{9}$... Seuls certains parmi eux se sont appliqués à relever dans le style même de Proust les procédés propres à " pratiquer » l'impressionnisme ainsi compris : comment transposer, à même les phrases, l'ordre de la perception afin qu'il conditionne semblablement l'ordre de la réception chez le lecteur $?^{10}$ Et pourtant, tel est bien le crédo de Proust, et sa devise en termes de style : « faire passer une impression en expression ».

La controverse essentielle de la question - et les malentendus qui peuvent en dériver -, tient assurément à la variété des acceptions dont le terme même d' ' impressionnisme » s'est peu à peu trouvé dépositaire, du domaine pictural au champ psychologique, du rayon thématique au faisceau de procédés formels. Essentiellement, le rapprochement a suscité deux attitudes critiques : celle qui consiste à dériver l'impressionnisme littéraire de l'impressionnisme pictural, sur la base d'une hypothétique transposition de procédés (tels que la « peinture » ou description par petites touches, la préférence de la couleur au dessin, le choix d'une palette claire, les scènes de plein air, le tremblé des contours, le refus de précision dans le détail, la préférence pour des sujets prosaïques et quotidiens etc.). Et par ailleurs, celle qui consiste à rattacher tant la peinture que l'écriture (romanesque ou poétique) à une commune racine inspiratrice : le privilège accordé à l'instant particulier, à sa saillance et sa surprise, à son caractère fugace et mouvant, à l'emprise immédiate du temps

8. Ferdinand Brunetière, «L'impressionnisme dans le roman » (1879); Le Roman naturaliste, Calmann-Lévy, 1883, $1896:$ 84-85.

9. Bally, Charles, «Impressionnisme et grammaire », Mélanges d'histoire littéraire et de philologue offerts à Bernard Bouvier, Genève, Société anonyme des éditions Sonor, 1920 :261-279, repris dans El impresionismo y el linguaje, Buenos Aires, Facultad de filosofia y letras de la Universidad de Buenos Aires, 1936, 1942 ; Mose, Ruth, L'Impressionnisme français : peinture, littérature, musique, Genève, Droz, 1952.

10. Suite à Leo Spitzer, « Le style de Marcel Proust » [1928], Études de style (tr. fr. Paris, Gallimard, « Tel », 1971), voir Michel Sandras, Proust ou l'euphorie de la prose (Paris, Honoré Champion, « Recherches proustiennes » $n^{\circ} 18,2010$ ). 
sur son devenir. Brunetière lança la première lignée dans cette étude sur Daudet, qui fit date. La seconde lignée se noue, en particulier, chez Jacques Dubois qui s'inspire de Maurice Cressot, culminera chez Henri Mitterand pour expirer chez Vouilloux ${ }^{11}$. Elle s'interroge sur la pertinence à identifier l'impressionnisme littéraire, ou mieux son « instantanéisme » ou «fragmentisme » (Dubois), à l' « écriture artiste ", à condition de ne pas en limiter le prototype aux seuls Goncourt, mais d'embrasser un « champ de dispersion » des stylèmes propre à rendre compte également d'auteurs tels que, non seulement Daudet et Loti, mais aussi Huysmans, Vallès, Cros, Laforgue ${ }^{12} \ldots$

Il a semblé utile de relire Proust à l'aune de $l^{\prime}$ « écriture artiste ${ }^{13}$ ainsi comprise, afin de vérifier la pratique d'une communauté de stylèmes en faisceaux convergents (I/ Impressionnisme scriptural). Mais une compréhension plus textuelle de l'impressionnisme, peut-être mieux rebaptisée « écriture de l'instantané ", fait ajouter aux procédés stylistiques au sens strict (souvent circonscrits à l'unité phrastique), des procédés formels de perspective et de découpage

11. Marcel Cressot, La Phrase et le vocabulaire de J.-K. Huysmans, Genève, Droz, 1938 ; Jacques Dubois, Romanciers français de l'instantané au XIXe siècle, Bruxelles, Palais des Académies, 1963; Henri Mitterand, «De l'écriture artiste au style décadent ", Histoire de la langue française 1880-1914, G. Antoine et R. Martin dir., Éditions du CNRS, 1985 : 467-477 ; Bernard Vouilloux, « L'« écriture artiste » : enjeux et présupposés d'un manifeste littéraire », Revue des Sciences Humaines, $\mathrm{n}^{\circ} 259$, « Les frères Goncourt », 2000 : 217-269 ; Id., « L'Impressionnisme littéraire' : une révision », Poétique, n 121, février $2000: 61-92$; Id., « Pour en finir avec l'impressionnisme littéraire. Un essai de métastylistique ", Questions de style, $n^{\circ}$ 9, $2012: 1-25$.

12. Le récent colloque Écrire en artistes : des Goncourt à Proust propose dans son argumentaire le paradigme ouvert suivant : Alphonse Daudet, les frères Goncourt, Francis Poictevin, Proust, Jean Lorrain, Huysmans, Marcel Schwob, Adolphe Retté, Rosny, Rodenbach, Jean Jullien, Descaves, Montesquiou, etc. (http:/:www.atilf.fr/spip.php?rubrique593\&idfirst=3768).Voir Pierre-Jean Dufief et Gabrielle Melisson-Hirschwald (dir.), Écrire en artistes : des Goncourt à Proust, Paris, Honoré Champion, 2016.

13. Une lecture est fournie par Luc Fraisse (« Du côté de chez Swann daté par sa prose d'art ? Les clochers de Martinville », in Dufief et Melisson-Hirschwald, op. cit.), qui reconnaît dans le morceau des clochers de Martinville un pastiche fort de l'écriture artiste. Mais l'analyse reste circonscrite à cet exemplaire, et comprise comme un pastiche plus ou moins volontaire et conscient du Proust de 1908 à 1913. Sans rien ôter à la pertinence de ces propos, fidèles à la question posée, l'approche pratiquée ici se donne un corpus plus étendu, une autre démarche et d'autres procédés d'analyse. 
descriptif, qui engagent à leur tour une cohérence d'écriture à un niveau plus compréhensif, interphrastique (II/ Impressionnisme structural). On confrontera cette comparaison critique (entre « écriture artiste » et style de Proust) au champ sémantique des termes phares en examen («impression », « impressionnisme », « impressionniste ») tels que permet de les cerner une étude immanente et inductive de leurs occurrences dans le corpus de la Recherche: on appréciera le fondement cognitif que lui confie Proust, comme posture d'accueil phénoménologique et geste d'appropriation du monde. En dialogue avec les rares critiques qui l'ont entendu de cette manière (dont Spitzer et Sandras), on voudra ensuite ébaucher une reconnaissance des procédés principalement syntaxiques voués, chez Proust, à faire passer l'impression avant l'intellection, à livrer au lecteur, dans l'ordre chronologique de la lecture ${ }^{14}$, $1^{\prime}$ impact des stimuli et sensations avant tout traitement de compréhension, d'élaboration, de classification et de restitution cognitive (III/ Impressionnisme cognitif). Cette perspective sémantico-syntaxique montre, pour l'ordre de livraison de l'information, les différents moyens syntaxiques employés par Proust afin de conférer à l'expression de la prise de conscience le même ordre que celui de sa saisie sensitive (impression - expression - raison). Enfin, un bilan éclairera la connivence profonde et fondamentale de l'impressionnisme proustien avec la disposition à la réminiscence (IV/ Impressionnisme mémoriel).

Le corpus de travail choisi se limite essentiellement à « Combray » ${ }^{15}$.

14. Que Proust ait conçu et pratiqué dans l'écriture sa propre «théorie » de la lecture est nouvellement argumenté en sciences cognitives par Maryanne Wolf, Proust and the Squid. The Story and Science of the Reading Brain, New York-London-Toronto-Sydney-new Delhi-Auckland, Harper Perennial, 2008.

15. La position de Proust par rapport aux poétiques de son temps est discutée, entre autres, dans Michel Sandras, Proust ou l'euphorie de la prose, op. cit. La teinte « artiste » des œuvres de jeunesse (en particulier Les Plaisirs et les Jours) est rappelée par Luc Fraisse dans « Du côté de chez Swann daté par sa prose d'art? Les clochers de Martinville » (Dufief et Gallisson-Hirschwald, op. cit.). Une saisie diachronique de l'évolution du style dans la Recherche est grandement compliquée par sa technique de composition en patchwork. D'où mon dévolu, modeste et prudent, quoique partiel et partial, sur « Combray » 1913 (cité à partir de l'édition d'À la recherche $d u$ temps perdu établie sous la direction de JeanYves Tadié, Paris, Gallimard, « Bibliothèque de la Pléiade », 4 vol., 1987-1989 ; t. IV: 286-295). 


\section{Impressionnisme scriptural : un faisceau de stylèmes}

À la suite des Goncourt eux-mêmes ${ }^{16}$, relayés par Brunetière, $l^{\prime}$ « écriture artiste » a tôt fait de fournir un « équivalent littéraire » de l'impressionnisme pictural. On en énumère un faisceau de stylèmes prototypiques, dont le noyau dur recueille tous les suffrages : un lexique enrichi d'archaïsmes, de néologismes et de mots rares, l'approximation dénominative et qualificative, par petites touches successives, une parataxe dépecée jusqu'à l'ellipse, le style substantif, et divers types de déplacements et de suspension tels que, dans le syntagme nominal, la montée des adjectifs en position gauche subjectivante, et dans la phrase, l'insertion de constructions détachées (appositives ou circonstancielles) entre le sujet et le verbe et entre le verbe et $\mathrm{l}^{\prime}$ objet $^{17}$. Ces quelques traits caractérisent de façon emblématique les écrits d'auteurs français tels que les frères Goncourt, Alphonse Daudet, Pierre Loti, J.-K. Huysmans, Jules Vallès et, avec plus de discrétion et de mesure " attique ", Flaubert, Maupassant, Zola ${ }^{18}$.

Considérés un à un, analytiquement, ces stylèmes apparaissent bien çà et là dans la Recherche, mais en vérité, ils ne manifestent pas une insistance telle qu'ils se fassent proprement voyants, sauf à les chercher à la loupe ${ }^{19}$ ou à se concentrer sur tel ou tel «morceau » aux senteurs de pastiche ou d'autopastiche ${ }^{20}$. Ou pour mieux dire, leur emploi est, partout ailleurs, si bien soumis au pro-

16. Dans leur célèbre préface aux Frères Zenmago.

17. Cette synthèse, quelque peu télégraphique, est redevable des apports de Charles Bally, Jacques Dubois, Henri Mitterand (voir note 11). Ce dernier établit une distinction singulière (en sous-classes) entre l'écriture artiste et le style décadent.

18. Jacques Dubois (op. cit.) insiste sur la divergence en deux branches de l'impressionnisme littéraire : l'un modéré et l'autre plus extrême.

19. Luc Fraisse 2016 (art. cité) souligne lui aussi le caractère discret de cette empreinte dans la Recherche : «Le détail du texte romanesque dissimule silencieusement, et véritablement sans crier gare, une assimilation des mots et même du style des deux frères [Goncourt] ».

20. Ce que proposent justement Michel Sandras (op. cit.) avec un morceau choisi de III, 911-912, et Luc Fraisse avec les clochers de Martinville. Une riche lignée critique intéressée au pastiche, genre magistralement pratiqué par Proust, s'attache tout particulièrement à celui du Journal des frères Goncourt incrusté dans le Temps retrouvé (pour une synthèse, voir Dictionnaire Marcel Proust, op. cit., sub voce). 
jet poétique de l'auteur que leur profonde nécessité les rend pour ainsi dire transparents, et non volontairement saillants, comme par contre dans le pastiche. Voyons quelques rapides exemples, tirés tour à tour des procédés lexicaux, syntaxiques, rhétoriques et actanciels.

LEXIQUE. Rien ne serait plus fallacieux que de trouver au lexique de Proust la moindre teinte « artiste ». Diverses grandes enquêtes lexicologiques ont montré la teneur essentiellement classique de son vocabulaire $^{21}$. La dernière en date a même réduit à portion infime la soi-disant néologie qu' on avait pu lui attribuer naguère faute de pouvoir enquêter tous azimuts sur les usages lexicaux de son temps, comme on peut le faire aujourd'hui grâce à l'Internet ${ }^{22}$. Et d'ailleurs, la tendance typiquement « artiste » à décliner les synonymes pour caractériser l'objet de discours par approximations successives semble bien éloignée du crédo proustien, selon lequel toute chose mérite une et une seule dénomination : la plus appropriée. Et si tel ou tel mot scientifique ou technique apparaît bien quelquefois dans l'une ou l'autre comparaison, il se trouve le plus souvent motivé par une volonté de contraste humoristique entre le prosaïsme de l'objet décrit et la spécialité de langue savante mise à contribution pour hausser d'un cran sa vision.

PARATAXE/hYPOTAXE. Rien ne serait moins vain non plus que de monter en épingle (en stylème véritable) les rares paragraphes paratactiques que peut présenter la Recherche, d'ailleurs souvent justifiés par le régime dialogal où ils s'insèrent, surtout lorsqu'il s'agit de « monologue intérieur » ou du moins d'endophasie (I, 4 et I, 7). C'est au contraire la superbe hypotaxe qui distingue notoirement ${ }^{23}$ le style de

21. Étienne Brunet, Le Vocabulaire de Marcel Proust, Paris, Honoré Champion, 1983 ; Sylvie Pierron, Ce beau français un peu individuel. Proust et la langue, Paris, Presses Universitaires de Vincennes, 2005.

22. Voir les enquêtes de Sylvie Bougeard-Pierron, "'Les mots nouveaux' de la Recherche. Néologie dérivationnelle », dans Geneviève Henrot Sostero et Isabelle Serça, Marcel Proust et la forme linguistique de la Recherche, Paris, Honoré Champion, « Recherches proustiennes » n. 30, 2013, pp. 221-238.

23. Pour rappel, quelques belles études sur la syntaxe de Proust se lisent chez Leo Spitzer, « Le style de Marcel Proust » art. cité ; Jean Milly, La Phrase de Proust, des phrases de Bergotte aux phrases de Vinteuil, Paris, Champion, « unichamp », 1983 ; Conrad Bureau, La Phrase de Proust. De la syntaxe fonctionnelle à l'analyse du style, 
Proust, telle qu'on a pu tour à tour la lui reprocher ou l'en féliciter. En voici un prototype, où, pour mieux éclairer la masse d'expansions qui gonflent la période, apparaissent en italique les constituants strictement nécessaires de la phrase («l'article... exprimait... le respect...») :

Dans le cas particulier, l'article du code à cause duquel il était peu probable que sauf le cas d'incendie Françoise allât déranger maman en présence de M. Swann pour un aussi petit personnage que moi, exprimait simplement le respect qu'elle professait non seulement pour les parents - comme pour les morts, les prêtres et les rois - mais encore pour l'étranger à qui on donne l'hospitalité, respect qui m'aurait peut-être touché dans un livre mais qui m'irritait toujours dans sa bouche, à cause du ton grave et attendri qu'elle prenait pour en parler, et davantage ce soir où le caractère sacré qu'elle conférait au dîner avait pour effet qu'elle refuserait d'en troubler la cérémonie (I, 29).

Une ossature toute simple ( $\mathrm{P}=$ un $\mathrm{SN}$ sujet « l'article du code », suivi d'un SN prédicat " exprimait le respect»), supporte des grappes d'expansions intérieures aux syntagmes fondamentaux : la détermination $\mathrm{du}$ " code » et les destinataires $\mathrm{du}$ " respect », ces derniers ferrés par un système corrélatif («non seulement, mais aussi »), une comparaison additive (« comme »), une énumération syndétique et la reprise lexicale (« respect qui ») : le régime du verbe tient sous une ferme convergence stylistique ${ }^{24}$, à lui tout seul, les deux tiers de la phrase. Ce type de construction expansive (déjà bien décrit par Spitzer, Bureau et Milly) suffit à expliquer la longueur de nombreuses phrases de Proust sans qu'interviennent à chaque fois d'éclatantes opérations de « démantèlement » (sauf à souligner, comme ici, l'insertion d'une comparaison entre tirets $\left.{ }^{25}\right)$. Ce qu'un tel type de phrase peut en revanche avoir en commun avec certaines pratiques d'écriture artiste, c'est la construction « en escalier » : mais, alors que

Thèse de doctorat de 3e cycle, Université de Provence, Lettres et Sciences humaines s.d. ; id., « Marcel Proust ou le temps retrouvé dans la phrase », Linguistique fonctionnelle et stylistique objective, Paris, P.U.F., 1976, pp. 178-231 ; Yvette Louria, La Convergence stylistique chez Proust, Paris, Nizet, 1971 ; et plus récemment, chez Isabelle Serça et Geneviève Henrot (éds.), Marcel Proust et la forme linguistique de la Recherche, op. cit.

24. Concept syntaxico-stylistique développé par Yvette Louria, op. cit.

25. Pour une fine analyse des parenthèses dans la phrase de Proust, voir Isabelle Serça, Les Coutures apparentes de la Recherche, Paris, Honoré Champion, « Recherches proustiennes », $\mathrm{n}^{\circ} 15,2010$. 
sa pratique «artiste » a pour but d'enclencher une dérive thématique d'apparence aléatoire, à l'instar du vagabondage du regard lui-même (du genre $\mathrm{Ab}=>\mathrm{Bc}=>\mathrm{Cd}=>\mathrm{De}$, etc.), il est bien plus fréquent que chez Proust, la fausse dérive (son pouvoir momentanément digressif) revienne en boucle exactement à son point de départ, comme ci-dessus, avec « cérémonie ». Ou bien elle agit, non point en dérive aléatoire et capricieuse du regard (en zigzag imprévisible), mais en ferme perspective de zoom, qui « file » soudain vers un gros plan inattendu, comme lorsque le regard mental du héros, parti du haut de sa chambre, plonge sur la place de Combray, s'insinue dans la boutique et l'arrière-boutique de l'épicier et s'écarquille sur les mains que ce dernier frotte énergiquement l'une contre l'autre :

De ma chambre je savais exactement la couleur qu'avait le soleil sur la place, la chaleur et la poussière du marché, l'ombre que faisait le store du magasin où maman entrerait peut-être avant la messe dans une odeur de toile écrue, faire emplette de quelque mouchoir que lui ferait monter, en cambrant la taille, le patron qui, tout en se préparant à fermer, venait d'aller dans l'arrière-boutique passer sa veste du dimanche et se savonner les mains qu'il avait l'habitude, toutes les cinq minutes, même dans les circonstances les plus mélancoliques, de frotter l'une contre l'autre d'un air d'entreprise, de partie fine et de réussite (I, 64).

Distensions et ReTARDEMENTS. Ceci dit, Proust bouscule lui aussi, bien souvent, l'ordre « canonique » de la phrase, par des insertions de corps étrangers au noyau argumental minimal : syntagmes appositifs, épithètes détachées, circonstants plus ou moins envahissants, incises, incidentes et parenthèses. Ce trait ressemble en effet au démantèlement pratiqué par l'« écriture artiste », qui éloigne volontiers, elle aussi, le sujet de son verbe et le verbe de son objet:

Mon corps, trop engourdi pour remuer, cherchait, d'après la forme de sa fatigue, à repérer la position de ses membres $(\mathrm{I}, 6)$.

On avait inventé, pour me distraire les soirs où on me trouvait l'air trop malheureux, de me donner une lanterne magique dont, en attendant l'heure du dîner, on coiffait ma lampe (I, 9).

Il se contenta, par un geste qui lui était familier chaque fois qu'une question ardue se présentait à son esprit, d'essuyer ses yeux et les verres de son lorgnon (I, 15). 
Sous l'espèce d'incognito qui lui faisait chez nous ce nom de Swann, ils hébergeaient - avec la parfaite innocence d'honnêtes hôteliers qui ont été chez eux, sans le savoir un célèbre brigand - un des membres les plus élégants du Jockey-Club (I, 15).

On peut fréquemment observer que ce report vers l'extrême droite de l'objet qui constitue l'apport rhématique de la phrase, par l'intercalation de nombreux éléments retardants (dont les circonstants), est régulièrement appliqué aux phrases qui aboutissent ainsi sur le détail descriptif saillant, la « pointe » d'humour, comme le remarquait déjà Spitzer en 1928.

Place DE L'ADJeCtif. Dans l' « écriture artiste », l'insertion dans la phrase de multiples syntagmes non argumentaux a pour corollaire, au palier inférieur du syntagme lui-même, la migration fréquente et parfois massive des adjectifs de la position droite à la position gauche, promise à une valeur subjectivante. On trouvera bien, chez Proust, au détour d'une page, telle tournure à la saveur " artiste ", comme ce printemps des pommiers avec « les timides bouquets de leurs rougissants boutons » (I, 144). Mais si le style de Proust n'hésite pas à exploiter l'apport qualifiant de l'adjectif (son morceau de bravoure en la matière reste, à ma connaissance, la chambre de tante Léonie et ses odeurs, morceau qui amasse jusqu'à 29 adjectifs en 12 lignes), il le fait d'une autre manière, qui n'insiste pas sur son déplacement « artiste », c'est-à-dire sur sa montée insistante vers la gauche du noyau syntagmatique. Il y a à cela sans doute quelques raisons qui tiennent aux règles de la syntaxe et du rythme français, dont Proust s'accommode sans éprouver le besoin de les malmener. En effet, ses adjectifs s'attirent en chaînes parfois vertigineuses, longues jusqu'à cinq, six, sept occurrences :

Legrandin était « de ces hommes qui [...] apportent à leurs occupations positives soit une insouciance mêlée de fantaisie, soit une application soutenue, lointaine, méprisante, amère et consciencieuse (I, 66).

[...] dans le cristal successif, lentement changeant et traversé de feuillages, de vos heures silencieuses, sonores, odorantes et limpides (I, 87).

On sentait que les notions que l'artiste médiéval, et la paysanne médiévale avaient de l'histoire ancienne [...], ils les tenaient non des livres, 
mais d'une tradition à la fois antique et directe, ininterrompue, orale, déformée, méconnaissable et vivante (I, 149).

Or, ces adjectifs sont le plus souvent coordonnés ; ils se modalisent d'adverbes (« lentement»), ou bien les participes présents ou passés («traversé ») enrôlent avec eux leur actance propre (« de feuillage », I, 87) : autant de raisons qui bloquent grammaticalement le passage à la position gauche de l'adjectif, une fois qu'il s'est développé en syntagme adjectival comme en I, 87.

Par ailleurs, le volume constitué par ces longues suites incite Proust, sensible à la respiration de la phrase, à respecter la cadence majeure, qui repousse vers la droite le segment le plus volumineux en termes de syllabes. Enfin, l'originalité des caractères exprimés par les adjectifs, porteuse de la vision personnelle accordée au réel, gagne en force si ceux-ci occupent dans la phrase la place qu'un suspens calculé permet de faire sonner en dernier, à la fin de la période, comme un feu d'artifice :

Sur la droite, on apercevait par-delà les blés, les deux clochers ciselés et rustiques de Saint-André-des-Champs, eux-mêmes effilés, écailleux, imbriqués d'alvéoles, guillochés, jaunissants et grumeleux, comme deux épis (I, 144).

RHÉTORIQUE. Le prétendu « chaos » (Spitzer) de l'énumération adjectivale chez Proust, qui fait les délices et la surprise du lecteur, présente en vérité une logique seconde que permettent de mettre au jour quelques principes de sa poétique : l'union fondamentale de tous les sens dans l'appréhension de l'objet suscite des qualifications synesthésiques ${ }^{26}$, qui associent expressément à la vue des notations olfactives, tactiles, gustatives, comme l'odeur « médiane, poisseuse, fade, indigeste et fruitée du couvre-lit à fleurs de tante Léonie » (I, 50). Ou bien, du moment que l'inspiration analogique tire ses motifs du contexte (c'est son ressort métonymique), l'hypallage vient intervertir les qualifications, donnant au son de la clochette du jardin de Combray les traits de Swann (l'ovale de son visage et l'or de ses cheveux $)^{27}$, aux clochers, des allures d'épis dans les champs ou de

26. Sur la synesthésie chez Proust, une monographie : Marisa Verna, Le Sens du plaisir. Des synesthésies proustiennes, Peter Lang, 2013.

27. Voir Leo Spitzer, «Le style de Marcel Proust » art. cité et Isabelle Serça, «Vertus de l'hypallage ", dans Antoine Compagnon et Kazuyoshi Yoshikawa (éds, avec 
poissons sur la côte, de brioche le matin ou d'oreiller le soir ${ }^{28}$, ou bien, toujours à Combray, aux endives, une "précocité », à l'omelette, une "faveur », au bifteck, une largesse qui ne sont pas celles, banales, de la saison ou du marché mais celles de l'heure où est servi, par exception, le repas du samedi : « des endives précoces, une omelette de faveur, un bifteck immérité » (I, 109).

Mais l'apparente contradiction locale des adjectifs ainsi juxtaposés de «manière explosive » peut aussi relever d'un facteur temporel : chaque adjectif peut convoquer un moment différent de l'après-midi de lecture, tantôt sonore (quand le clocher sonne l'heure), tantôt silencieux (dans les intervalles), et leur juxtaposition signaler, par-delà l'instant, la durée que borne celui-ci. Ou bien elle signale par son incongruence même la superposition de deux époques, l'une passée (tel après-midi d'été à Combray), l'autre présente (telle matinée d'hiver à Paris) : " mes pas nostalgiques et profanateurs ", lilas « invisibles et persistants $» \ldots{ }^{29}$. À bien y regarder, l'association divergente des adjectifs n'a pas chez Proust, autant que chez les écrivains « artistes », la même motivation de fantaisie instantanée inspirée par des impressions débridées, volontairement désordonnées, mais relève au contraire (témoin les avant-textes) du ciment multiple auquel il confie «la solidité de ses moindres parties $»^{30}$.

Ainsi, le trait frappant qui motive et supporte l'analogie se cherche une place de choix, quitte à remonter d'un degré dans la hiérarchie syntaxique, comme dans la vision « bergère » de l'église de Combray :

Une église résumant la ville [...] tenant serrés autour de sa haute mante sombre, en plein champ, contre le vent, comme une pastoure ses brebis, les dos laineux et gris des maisons rassemblées (I, 47).

la collaboration de M. Vernet), Swann, le centenaire (Actes du Colloque de Cerisy-la-Salle, 27 juin-4 juillet 2012), Paris, Éd. Hermann, 2013 : 35-53.

28. Gérard Genette, «La métonymie chez Proust », dans Figures III, Paris, Le Seuil, 1972 : 41-69.

29. Voir Geneviève Henrot, Délits/Délivrance. Thématique de la mémoire proustienne, Padova, Cleup, «Letture e ricerche francesi » n. 4, 1991, p. 89-95 et « Le Fléau de la balance. Poétique de la réminiscence », Poétique, n 113 : 189-210.

30. Lettre du 2 août 1919 à Jean de Gaigneron, in Philippe Kolb, Lettres retrouvées, Paris, Plon, 1966. 
... où la montée de l'image (" dos laineux et gris ») par rapport au comparant («maisons ») concentre l'attention en même temps qu'elle compose un chiasme entre les deux isotopies (Isot.1 : église [Isot.2 : mante, pastoure, brebis, dos laineux et gris] Isot. 1 : maisons) et fait en sorte que la qualification « dos laineux et gris » se trouve à égale proximité de son pantonyme («brebis ») et de son comparé («maison ") ; l'image se trouve ainsi, grâce au chiasme syntaxique, embrassée par son comparé, lovée en creux, comprise entre ses bornes.

STYLE SUBSTANTIF. Cette «montée » syntaxique du trait descriptif en quête de saillance est commune à la focalisation méronymique (ici le dos des brebis pour les toits gris des maisons, puisque la vue est plongeante) et à la focalisation de l'action ou de la sensation qui mérite l'attention du lecteur, en tant qu'elle est la source la plus vive de l'impression reçue. Le défilé des soldats dans Combray met l'accent sur « l'éclat » de leurs casques sous le soleil (I, 85-86) ou sur la rapidité du passage des troupes : «Et le galop des chevaux rasait les maisons, couvrant les trottoirs submergés comme des berges qui offrent un lit trop étroit à un torrent déchaîné » (I, 87). Or, ce sont plutôt les chevaux qui rasent les maisons, que leur galop. Cette construction, qui fait que la qualité passe avant son objet, fait signe au style substantif attribué à $l^{\prime}$ « écriture artiste ». On en trouve pas mal d'exemples chez Proust, même associés au pluriel, comme ici : «mes parents voyaient les élégances régner dans son visage » $(\mathrm{I}, 19)$. Quelques autres occurrences :

Nous vîmes sur le seuil brûlant du porche le tumulte bariolé du marché (I, 123).

Le ciel entier que jonche la dispersion d'innombrables pétales soufrés ou roses $(\mathrm{I}, 129)$.

Une suite de chapelles qui disparaissaient sous la jonchée de leurs fleurs amoncelées en reposoir (I, 136).

Le visage de Théodore, enluminé de la rougeur d'une pomme mûre (I, 149).

Cependant, le naturel avec lequel ce stylème s'amalgame aux autres dans le style de Proust, au point de passer facilement inaperçu, tient à la prégnance perceptive qu'il garantit en contexte. La substantivation de la qualité accorde à la sensation à laquelle elle 
réfère une saillance plus apte à frapper le lecteur. Et cette saillance est justifiée tantôt par un punctum dont jouit l'intention réaliste du portrait (la nacre d'une peau, le tombant d'une paupière, la couperose d'une joue, le rebondi d'une croupe), tantôt par la métaphore qui s'amorce là ${ }^{31}$, tantôt encore par la nature exacte et précise des perceptions du sujet. Aussi verra-t-on le style substantif requis de préférence dans l'expression de l'impression, lorsqu'il s'agit de préciser quelle sensation particulière produite par l'objet en est la cause, parce que perçue en premier : l'éclat des casques des soldats (I, 8586), les égouttements gommeux de soleil (I, 64), le roucoulement de la colombe (I, 71), les pas de mes parents reconduisant Swann, le dessèchement des tiges de tilleul (I, 50), ou encore, le bruit léger de la robe de jardin qui passe dans le couloir (I, 19) ou enfin l'éventail vertical et prismatique des gouttelettes multicolores du tuyau d'arrosage (I, 138-139).

ACTANCE : promotion de la sensation, démotion du sujet (selon Guillaume) comme siège, théâtre de l'événement. Tout comme, au niveau micro-syntagmatique, le syntagme nominal place en tête la sensation-amorce et en complément déterminatif l'objet-source, de même, à un niveau supérieur, celui de la phrase, s'opère une montée en position sujet de ce même syntagme contenant la sensation, cependant que le sujet lyrique rétrograde en position dative ou circonstancielle : il devient bénéficiaire ou victime du coup de théâtre sensationnel, voire simplement siège, lieu, scène de ce même «événement $»^{32}$. C'est à quoi travaillent, entre autres, le choix des verbes d'une action attribuée aux sensations agents, la construction pronominale moyenne-passive de bien des phrases proustiennes ${ }^{33}$ et, sy-

31. Le "feston" des domestiques assis sur le trottoir amorce la comparaison au cordon d'algues sur la plage: "les domestiques ou même les maîtres, assis et regardant, festonnaient le seuil d'un liséré capricieux et sombre comme celui des algues et des coquillages dont une forte marée laisse le crêpe et la broderie au rivage, après qu'elle s'est éloignée » (I, 89).

32. Pour une lecture de l'impression comme événement, voir Geneviève Henrot Sostero, «La réminiscence comme événement : saillance, agentivité, transformation ", in Elio Ballardini, Roberta Pederzoli, Sandrine Reboul-Touré, Geneviève Tréguer-Felten (éds.), Les facettes de l'événement : des formes aux signes, mediAzioni 15, http://mediazioni.sitlec.unibo.it.

33. Geneviève Henrot Sostero, « Mémoire et voix moyenne ou comment dire l'involontaire », Bulletin d'Informations Proustiennes n 35, $2005:$ 77-92. 
métriquement, la démotion du sujet en milieu ou fin de phrase, impliquée par la promotion de la perception qui le frappe ou du sentiment qui l'envahit. Souvent cette « action » du réel sur le sujet anime de vie, de geste et de mouvement, voire personnifie, certains objets ou les sensations qui s'en dégagent:

Ces brillantes projections promenaient autour de moi des reflets d'histoire ancienne (I, 10).

Le petit village de Roussainville sculptait sur le ciel le relief de ses arêtes blanches (I, 148).

La petite porte de derrière de notre jardin [...] était venue avec le coin de la rue du Saint-Esprit nous attendre au bout de ces chemins inconnus (I, 114).

Une animation du paysage en mouvement caractérise aussi ce passage prototypiquement « impressionniste » des clochers de Martinville et quantité de descriptions ambulatoires motivées par le déplacement du promeneur, en calèche, en train, en voiture, en gondole.

\section{Impressionnisme structural : les formes de l'espace et du temps}

L'« écriture artiste » se caractérise, comme du reste la peinture impressionniste, par une saisie de l'espace qui se donne comme orientée, provenant d'un foyer individuel de perception ${ }^{34}$. Des prises de vue asymétriques abandonnent la construction classique autour d'une ligne de fuite centrale au tableau. Plongées et contre-plongées, cadres décentrés, écrans et barrières visuelles livrent un objet de vision incomplet et vu sous un angle insolite. Aussi le paysage, perdant la rigueur classique de la composition balancée autour d'un axe médian, se présente-t-il plutôt comme un fragment de paysage, une "vue " partielle et partiale, comme découpée artificiellement par le cadre du tableau. Contemporain du pré-cinéma et d'instruments d'optique fantaisistes, l'impressionnisme pictural semble revendiquer la pro-

34. Étymon spirituel : dissocier l'objet en soi et sa saisie subjective par un point de vue particulier. D'où situer un foyer de la vision, un cadrage particulier qui échappe aléatoirement à la composition classique organisée sur la ligne de fuite centrale orientée vers l'horizon (Jean Clay). 
priété exclusive de chaque "vue », comme l'enfant de la Recherche imaginait que pouvait être le théâtre :

Je n'étais pas éloigné de croire que chaque spectateur regardait comme dans un stéréoscope un décor qui n'était que pour lui, quoique semblable au millier d'autres que regardait, chacun pour soi, le reste des spectateurs (I, 72).

On n'aura nulle peine à reconnaître ce que la description proustienne partage en effet avec cette poétique du fragment, comme il apparaît aussi dans l'étude de ses portraits ${ }^{35}$. Cerné et délimité par le cadre d'une fenêtre, d'un vasistas, d'un hublot, d'une vitre de train ou d'un œil-de-bœuf ${ }^{36}$, le paysage se débite en copeaux tronqués et biaisés : tantôt plongée curieuse de tante Léonie sur la place de Combray, tantôt contre-plongée anxieuse du jaloux vers une façade éclairée, tantôt survol rêveur des cheminées de Venise ${ }^{37}$ ou des toits de Paris :

Je sais une fenêtre où on voit après un premier, un second et même un troisième plan fait par les toits amoncelés de plusieurs rues, une cloche violette parfois rougeâtre, parfois aussi, dans les plus nobles « épreuves » qu'en tire l'atmosphère, d'un noir décanté de cendres, laquelle n'est autre que le dôme de Saint-Augustin (I, 65).

Cette vue sur les toits de Paris présente plusieurs traits revenant aussi dans la fragmentation artiste des paysages : le découpage insolite de la ville par le haut (rien que des toits) et, partant, son caractère prosaïque, qui n'attend point de l'objet, mais du style, sa qualité esthétique ; le point de vue orienté à partir d'une fenêtre bien précise ; la variation de la lumière qui en fournit des clichés différents, violet, rouge ou noir ; et enfin une construction en énigme, qui retarde jusqu'au bout de la phrase le pantonyme identifiant l'objet peint. La

35. Voir pour une synthèse, l'article "Portrait", dans Dictionnaire Marcel Proust, Paris, Honoré Champion, 2003, sub voce.

36. Le narrateur adulte projette sur tout paysage les mêmes conditionnements qui furent ceux de la lanterne magique de son enfance : « [...] le château était découpé selon une ligne courbe qui n'était autre que la limite d'un des ovales de verre ménagés dans le châssis qu'on glissait entre les coulisses de la lanterne. Ce n'était qu'un pan de château et il avait devant lui une lande où rêvait Geneviève qui portait une ceinture bleue » $(\mathrm{I}, 9)$.

37. On sait aussi la part de voyeurisme que telles postures enclenchent ou révèlent. 
vue n'est pas donnée, éternelle et universelle, mais construite, particulière et momentanée, propre à un instant du jour.

Ce fragmentisme de la vision imprègne aussi les premières pages traitant de la mémoire : non celle, vaine et impuissante, de la mémoire de l'intelligence, ni encore la mémoire involontaire, mais ce type intermédiaire né des réveils nocturnes, qui a fixé "Combray à sept heures du soir » en un seul pan de mur :

Je n'en revis jamais que cette sorte de pan lumineux découpé au milieu d'indistinctes ténèbres. Pareil à ceux que l'embrasement d'un feu de Bengale ou quelque projection électrique éclairent et sectionnent dans un édifice dont les autres parties restent plongées dans la nuit (I, 43).

Or, si le fragment d'espace s'impose au regard du narrateur, ce n'est pas que celui-ci soit disposé à s'en satisfaire : suivant la leçon du curé de Combray qui conseillait une vue « combinée » du paysage de Combray, à la fois du haut du clocher et du dédale des rues, de même le voyageur en train s'acharne à « rentoiler » au pas de course les deux vues divergentes du côté nuit et du côté aurore dont la rame du train elle-même semble être la ligne de partage.

FRAGMENTS D'INSTANTS. Sujette à un éclairage particulier, fugitif et labile comme cet embrasement bengali, la vision proustienne, tout comme la vision impressionniste, est tributaire de l'instant. La frise temporelle de la durée se découpe comme celle de l'espace en tronçons isolés, plus ou moins successifs, pour mieux montrer encore ce qu'a d'irréductible et de dépaysant tel jeu de lumière et de couleurs sur l'uniformité d'un même paysage. Il y aurait tout un livre à écrire sur la lumière chez Proust, le modelé qu'elle donne aux objets et la scansion qu'elle révèle du temps (aurores et clairs de lune, rais d'éclairage qui s'allument ou s'éteignent). Mais linguistiquement parlant, le parcours stylistique des procédés impressionnistes mérite qu'on s'attache à la notion linguistique $\mathrm{d}^{\prime}$ « aspect ».

Car c'est à l'aspect et à ses diverses réalisations linguistiques (adverbes temporels, préfixes inchoatifs ou infixes progressifs des verbes, auxiliaires ventif et andatif, sécants, ingressifs, progressifs ou égressifs... $)^{38}$ que Proust confie le soin de borner l'instant décrit

38. Marc Wilmet, Grammaire critique du français, Bruxelles, De Boeck-Duculot, 20105, sub voce. 
entre « encore » et « ne plus », entre "s'endormait » et "pâlissait», entre "venait de » et "allait», dans des suites de participes présents figeant l'action, le temps de la pose : ainsi de l'épicier du dimanche qui «venait d'aller passer sa veste » et «se préparait à fermer » (voir plus haut), ou de Mme Sazerat qu'on surprend à l'église dans un furtif geste de piété :

On voyait s'agenouiller un instant Mme Sazerat, posant sur le prieDieu voisin un paquet tout ficelé de petits fours [...] qu'elle venait de prendre chez le pâtissier d'en face et qu'elle allait rapporter pour le déjeuner (I, 59).

Plus « Manet » encore, ces asperges en métamorphose dans l'arrière-cuisine de Françoise, au pied «encore souillé pourtant du sol de leur plant », qui « laissaient apercevoir en ces couleurs naissantes d'aurore, en ces ébauches d'arc-en-ciel, en cette extinction de soirs bleus, cette essence précieuse... » (I, 118). À Paris, le garçon lit des " affiches encore humides et boursouflées de colle » et surprend (I, 72) chez son oncle « une jeune femme qui achevait de manger une mandarine » $(\mathrm{I}, 75)$.

La vérité de l'instant s'explique parfois par sa position dans un algorithme naturel (de croissance, par exemple, ou de parcours), tel qu'il est possible de faire se côtoyer, en raccourci, quelques coupes successives, pour mieux saisir le changement: des fleurs de tilleul toutes fraîches puis desséchées : "Cette flamme rose de cierge, c'était leur couleur encore, mais à demi éteinte et assoupie, dans cette vie diminuée qu'était la leur maintenant et qui est comme le crépuscule des fleurs » (I, 51); ou bien des lilas en pleine floraison, puis fanés :

Le temps des lilas approchait de sa fin ; quelques-uns effusaient encore en hauts lustres mauves les bulles délicates de leurs fleurs, mais dans bien des parties du feuillage où déferlait il y avait seulement une semaine leur mousse embaumée, se flétrissait diminuée et noircie une écume creuse, sèche et sans parfum $(\mathrm{I}, 134)$.

Mainte description proustienne est ainsi dès l'abord aspectualisée afin de cerner au plus près l'infinitésimal instant de la vision, dans l'éclair fugace du rayon spécial qui l'illumine, « reflet immobile 
comme un papillon posé » (I, 82) (c'est le moment de l'analyse) et ensuite prise dans un mouvement de variation du même ou quasimême qui sertit cet instant dans une chaîne d'autres instants semblables, afin de recomposer le ruban de la durée (c'est le moment de la synthèse) : ainsi des divers clochers de Combray, des mers de Balbec, ou encore des différentes phases du couchant dans ces retours de promenade tantôt « au commencement de la saison », tantôt « dans l'été », tantôt encore " certains jours forts rares », jusqu'à l'automne ${ }^{39}$. Loin d'exacerber l'étanchéité absolument monadique du pur instant, Proust n'a de cesse de le réintégrer dans une série, dont la solidité restitue la durée : ce « film » de la durée, tressautant comme Golo dans la lanterne magique, est l'équivalent temporel $\mathrm{du}$ « rentoilage » de la vision spatiale. On comprend que Proust ait pu insister sur la primauté, chez lui, de la vision sur la technique.

\section{Impressionnisme cognitif : primauté de la perception première}

Pour comprendre laquelle des lignées impressionnistes s'applique le plus pertinemment à Proust, et quel heur peut avoir chez lui le rapprochement entre « impressionnisme » et « écriture artiste », c'est en effet à Proust lui-même qu'il conviendrait d'abord de demander quelle extension sémantique a pour lui, moins le mot « impressionnisme » (quatre rares occurrences dans la Recherche), que sa racine, voire son étymon spirituel, $\mathrm{l}^{\prime}$ « impression ». Ce mot dont Proust fait souvent usage mérite d'ailleurs que soit discernée avec une précision toute linguistique l'aire sémantique de son emploi dans la Recherche. Des 383 occurrences d'« impression » relevées dans la Recherche, il convient de séparer des emplois substantifs pleins ceux que commande une construction figée du genre locution prépositionnelle (« sous l'impression de ») ou verbe support : soit l'« impression» constitue le régime d'une action exercée par un objet du monde (« causer/ donner/ produire/ faire une impression »), soit son sujet focalise plutôt le siège perceptif (« avoir/ éprouver/ goûter/ recevoir une impression »). Quant aux emplois substantifs pleins, leur environnement gauche penche pour une assiette présentatoire

39. Voir l'analyse qu'en fait Michel Charles dans son Introduction à l'étude des textes, Paris, Seuil, « Poétique », 1995: 148 et ss. 
(Damourette et Pichon) inconnue, nouvelle (" une ", « une certaine », «quelque ») ou plus rarement pour une relation possessive («mon », « nos », « cette » par anaphore). Quant à ses expansions droites, elles se partagent une qualification où domine une " prosodie sémantique » du mystère, de l'énigme, de l'indéchiffrable («nouvelle», « étrangère », « obscure», « confuse »), de la fugacité ( «fragile », « éphémère »), de l'intensité («forte », « pleine », " précieuse », « définitive »). Les épithètes relevant du champ du sentiment («triste », « douloureuse », « despotique », « mélancolique », " bienheureuses») s'accordent avec un emploi dominant de la construction « impression de + Sentiment », par laquelle il apparait bien que le phénomène relève entièrement du ressenti affectif (« impression d'ennui, de dégoût, de désordre, d'isolement, d'horreur, de charme, de tristesse, de fatigue »). Les cas restants de la construction en « de » relèvent de la détermination spatiale (« impressions de » Doncières, de Venise, de Balbec, de Combray), ou temporelle (« impressions anciennes, passées, d'autrefois, de la veille, contemporaines, suivantes, du lendemain »), ou encore identificatrice (« impression de mobilité, vie, vérité, mensonge, du temps qu'il faisait »).

Les acceptions vérifiées à travers les faisceaux sémiques actualisés en contexte voient dominer les occurrences localisées vers la fin du roman, où « impression » entre en résonance avec « expression » et avec « réminiscence ». L'impression proustienne relève d'une expérience cognitive impromptue, dont il s'agit d'« approfondir » $l^{\prime}$ origine afin de $l^{\prime}$ « exprimer » :

Or la recréation par la mémoire d'impressions qu'il fallait ensuite approfondir, éclairer, transformer en équivalents d'intelligence, n'était-elle pas une des conditions, presque l'essence même de l'art? (IV : 349).

Tout comme les écrivains et peintres impressionnismes, Proust aime fixer l'impression fugitive : le frétillement d'un rayon de lumière, la fibrillation d'une aile de papillon, le frisson d'un lacis de feuilles ensoleillées. Mais, à la différence des écrivains " artistes ", Proust ne peut se contenter de l'instantané en soi et pour soi, tant sa vocation de philosophe le pousse à tirer de ces clichés autant de lois sur le vivant. Néanmoins, pour garder bien distinctes d'une part, la 
source de l'impression et d'autre part, son traitement par la raison, il s'ingénie à grossir l'intervalle de la réflexion, à le retarder, à l'éloigner de son amorce. Comme à le gloser à coups de comparaisons : il compare le contretemps entre impression et intellection, comme entre sentiment et raison, au désaccord entre le tonnerre et l'éclair. Un décalage se creuse entre l'immédiateté de l'impression et le laborieux retard que prend sur elle son élaboration par la pensée : le sujet, assailli par une ou plusieurs sensations qui provoquent instantanément chez lui une forte impression, devient le théâtre d'une intense participation à la vision, où n'interviennent dans l'immédiat ni sa volonté, ni sa maîtrise, ni sa raison :

C'est l'inverse de ce soulagement que j'éprouvais quand mon chagrin entrait en moi d'une façon infiniment plus rapide, presque instantanée, à la fois insidieuse et brusque, par l'inhalation - beaucoup plus toxique que la pénétration morale-, de l'odeur de vernis particulier à cet escalier (I, 28).

Proust engage à cet effet plusieurs procédés de retardement qui invitent le lecteur à suspendre avec lui le diagnostic de la raison. Ajoutés aux détachements déjà rencontrés en (I) et aux qualifications centrifuges, interviennent divers procédés de suspens, tels que l'énigme, la suspension syntaxique : le portrait de Françoise, la pluie qui tombe. L'énigme descriptive pure est rare, mais inoubliable :

À peine arrivions-nous dans l'obscure antichambre de ma tante que nous apercevions dans l'ombre, sous les tuyaux d'un bonnet éblouissant, raide et fragile comme s'il avait été de sucre filé, les remous concentriques d'un sourire de reconnaissance anticipée. C'était Françoise, immobile et debout dans l'encadrement de la petite porte du corridor comme une statue de sainte dans sa niche (I, 52).

Le second exemple joint au retard du thème-titre la parataxe de phrases nominales, la succession de participes présents et l'accumulation d'adjectifs, elles aussi typique du style artiste :

Un petit coup au carreau, comme si quelque chose l'avait heurté, suivi d'une ample chute légère comme de grains de sables qu'on eût laissés tomber d'une fenêtre au-dessus, puis la chute s'étendant, se réglant, adoptant un rythme, devenant fluide, sonore, musicale, innombrable, universelle : c'était la pluie (I, 100). 
Un effet semblable est produit par l'architecture syntaxique, lorsque celle-ci est maniée pour distendre le temps qui sépare l'impression pure de sa compréhension (tout comme l'impression première de sa réminiscence) :

Mais au lieu de la simplicité, c'est le faste que je mettais au plus haut rang, si, après que j'avais forcé Françoise, qui n'en pouvait plus et disait que les jambes « lui rentraient», à faire les cent pas pendant une heure, je voyais enfin, débouchant de l'allée qui vient de la porte Dauphine image pour moi d'un prestige royal, d'une arrivée souveraine telle qu'aucune reine véritable n’a pu m'en donner l'impression dans la suite, parce que j'avais de leur pouvoir une notion moins vague et plus expérimentale - emportée par le vol de deux chevaux ardents, minces et contournés comme on en voit dans les dessins de Constantin Guys, portant établi sur son siège un énorme cocher fourré comme un cosaque, à côté d'un petit groom rappelant le «tigre " de «feu Baudenord ", je voyais - ou plutôt je sentais imprimer sa forme dans mon cœur par une nette et épuisante blessure - une incomparable victoria, à dessein un peu haute et laissant passer à travers son luxe « dernier cri » des allusions aux formes anciennes, au fond de laquelle reposait avec abandon Mme Swann $[\ldots]^{40}$.

Plus encore que dans les « énigmes » précédentes, ce dernier passage exploite cette distension entre impression et identification pour y instiller la saisie scripturale des « remous concentriques » de l'impression toute fraîche. L'impression de « faste » est portée en tête de phrase par l'extraction (" c'est... que »). Suit le déploiement minutieux des circonstances temporelles («après que, pendant une heure, enfin ») et spatiales («l'allée qui vient de la porte Dauphine»), qui encadre le sens impliqué (la vue). Cependant, l'objet de la perception (le C.O.D. de « je voyais »), se trouve indéfiniment retardé par l'antéposition de syntagmes à fonction d'épithètes détachées construits autour de participes (« débouchant ", « emportée », " portant »), à leur tour expansés de leurs arguments et de comparaisons (la reine

40. Rokus Hofstede, traducteur de la Recherche en néerlandais, exposait au colloque Traduire Du côté de chez Swann (Padoue, Italie, 14 et 15 novembre 2013) les acrobaties syntaxiques qu'impose le néerlandais pour disposer les données de la perception dans le même ordre logique et chronologique que l'original proustien. Voir « Parti pris d'une retraduction néerlandaise de Du côté de chez Swann (II). Questions de syntaxe ", in Geneviève Henrot Sostero et Florence Lautel-Ribstein, Traduire À la recherche du temps perdu de Marcel Proust, Paris, Classiques Garnier, « Revue d'études proustiennes » n 1, 2015 : 357-363. 
véritable, les dessins de Guys, le cosaque), au point que soit sentie comme utile à la mémoire du lecteur la répétition du verbe « je voyais ». Cette répétition a également comme fonction d'annuler le temps de lecture de ces circonstances, montrant leur « prise instantanée », telle que vient la gloser une rectification entre tirets : « ou plutôt je sentais imprimer sa forme dans mon cœur ». Significative d'un ordre perceptif, la progression bien scandée du regard qui saisit d'abord le cadre, puis l'avant du véhicule par ordre décroissant d'évidence ou de saillance (les chevaux, le cocher, le groom), le véhicule lui-même, et enfin l'objet véritable de l'attente, au fond de la voiture : Mme Swann, en clausule.

Il apparaît donc clairement que l'acception privilégiée confiée par Proust au terme s'explique, en contexte cognitif phénoménologique, comme la trace laissée par une perception à travers les sens, antérieure à toute élaboration par la pensée. Dans la même mouvance, l'impression (souvent accompagnée de l'adjectif " première », désigne alors aussi l'expérience sensitive et affective qui va se loger dans un souvenir oblitéré, et que seule une sensation identique sortira de son «vase » soudain descellé, sous forme de réminiscence ${ }^{41}$.

\section{Impressionnisme mémoriel : de l'impression à la réminis- cence}

Aussi n'y a-t-il guère lieu de s'étonner que l' « impressionnisme » chez Proust trouve à s'exprimer au mieux dans ces épisodes qui rendent le mieux compte de l'impressionnabilité de son esprit. La mémoire involontaire, en particulier, est l'application par antonomase de l'impressionnisme proustien, où l'écart entre le premier et le second geste d'appréhension du réel (impression - réminiscence) est distendu jusqu'à l'extrême, non plus de quelques secondes habilement gonflées pour les besoins de la démonstration, mais des années, voire toute une vie. La nature même du phénomène psychique s'accommode spontanément des procédés stylistiques et formels reconnus à l'écriture artiste : ainsi, une assomption si prégnante de la

41. Cette dominance de l'acception mémorielle du mot «impression » transparaissait déjà dans mon "À propos d'impressions. Petite mise au point », Bulletin Marcel Proust, 1999, n 49, 1999 : 137-146. 
sensation au détriment de l'objet qui l'amorce s'accommode bien de la substantivation du caractérisant : j'ai montré ailleurs cette sélection fine et précise du trait sensitif sur lequel repose le rappel inattendu du souvenir ${ }^{42}$, tel le sifflement des trains dans la campagne (I, 3).

On verra aussi s'y appliquer divers autres procédés de style recensés comme « artistes » : au niveau lexical, la désignation progressive, et la motivation cachée des suites adjectivales ; au niveau syntaxique, le jeu alterné de mise en suspens et de mise en avant ; au niveau actanciel, la réorganisation du scénario agentiel au profit de la sensation et du souvenir ; au niveau cognitif, le déploiement holistique du souvenir. Je n'en donnerai ici que quelques exemples, ayant déjà développé ailleurs ces fonctionnements stylistiques de la mémoire.

C'est dans l'expression de la réminiscence que la technique du suspens syntaxique trouve son application la plus fonctionnelle, la plus « poïétique ». J'ai décrit ailleurs l'équilibre structural qui s'instaure entre le développement de l'impression première et celui de la réminiscence, et qui s'appuie sur un jeu symétrique de rejet en fin de phrase, suivi d'une promotion de la sensation impliquée ${ }^{43}$. Mais sans aller chercher bien loin, l'épisode de la madeleine illustre de façon éclatante le suspens syntaxique, procédé qui consiste à rejeter en fin de phrase, à coup d'insertions circonstancielles, l'objet de l'impression chargé de déclencher la sensation-amorce :

Il y avait déjà bien des années que, de Combray, tout ce qui n'était pas le théâtre et le drame de mon coucher n'existait plus pour moi, quand, un jour d'hiver, comme je rentrais à la maison, ma mère, voyant que j'avais froid, me proposa de me faire prendre, contre mon habitude, un peu de thé (I, 44).

Dans le même épisode, la désignation progressive de l'objet se donnera le loisir d'avancer par approximation, du générique au spécifique, de l'indéfini au défini. Voici quelques maillons seulement de la chaîne de référence qui amène peu à peu le souvenir, retardant de deux pages sa nomination véritable, «tout Combray » :

42. Geneviève Henrot, « Le Fléau de la balance », art. cité.

43. Ibidem. 
[...] je sens tressaillir en moi quelque chose qui se déplace, voudrait s'élever. Quelque chose qu'on aurait désancré à une grande profondeur; je ne sais ce que c'est, mais cela monte lentement [...]. Ce qui palpite ainsi au fond de moi, ce doit être l'image, le souvenir visuel, qui, lié à cette saveur, tente de la suivre jusqu'à moi $[\ldots](\mathrm{I}, 45)$.

Le même passage permet encore de montrer en acte le jeu de promotion de la sensation (sa position fonctionnelle de sujet) et de démotion du sujet lyrique, devenu siège de l'événement (« en moi »), que le Narrateur glose en ces termes : « Grave incertitude toutes les fois que l'esprit se sent dépassé par lui-même ; quand lui, le chercheur, est tout à la fois le pays obscur où il doit chercher, et où tout son bagage ne lui sera de rien » (I, 45).

Parcourir tout le corpus de réminiscences qui parsèment la Recherche (cent motifs) m'a permis naguère de montrer combien la « logique cognitive » de la mémoire involontaire informait la syntaxe en faisant fréquemment recours, entre autres, à la voix moyenne ${ }^{44}$, afin qu'à la sensation, ou au souvenir, soient réservés les feux de la rampe, afin que l'esprit les fasse enfin « entrer dans sa lumière » (I, 45). Et là, un dernier stylème « artiste » vient servir à propos la poétique de la réminiscence, surtout quand celle-ci déploie la résurrection du passé dans toutes ses coordonnées : c'est l'accumulation. La parataxe, asyndétique d'abord, puis polysyndétique, projette encore une fois en clausule la « tasse de thé » et marque ce final de « Combray I, 1 », par quoi le roman démarre vraiment :

Et comme dans ce jeu où les Japonais s'amusent à tremper dans un bol de porcelaine rempli d'eau, de petits morceaux de papier jusque-là indistincts qui, à peine y sont-ils plongés, s'étirent, se contournent, se colorent, se différencient, deviennent des fleurs, des maisons, des personnages consistants et reconnaissables, de même maintenant toutes les fleurs de notre jardin et celles du parc de M. Swann, et les nymphéas de la Vivonne, et les bonnes gens du village et leurs petits logis et l'église $e t$ tout Combray et ses environs, tout cela qui prend forme et solidité est sorti, ville et jardins, de ma tasse de thé (I, 47).

À la curiosité de savoir si l'impressionnisme personnel ainsi cerné et détaillé rencontre dans sa mise en texte les procédés typiques

44. Geneviève Henrot, «Mémoire et voix moyenne », art cité. 
reconnus comme constitutifs de l'écriture artiste, on a pu observer qu'un faisceau cohérent de traits stylistiques se manifeste en effet dans l'écriture d'un ressenti instantané, mais que celle-ci se voue davantage à l'expression d'un événement intérieur, de nature affective, déclenché par une amorce extérieure aussi ténue que pointue et pénétrante. Par ailleurs, l'utilisation conditionnelle qu'en fait l'écrivain, subordonnée à l'expression d'une expérience cognitive immédiate (l'impression « première ») ou médiatisée par la mémoire (la réminiscence) l'empêche d'apparaître, par sa saillance, comme un " procédé » exhibé pour sa surprise même. En ce sens, la discrétion des stylèmes, totalement ancillaires du projet poétique, revient à apprécier une fois de plus le « classicisme » du style de Proust. Ou son sobre «fonctionnalisme».

Université de Padoue 